che I promessi sposi non sono affatto il romanzo dell'idillio e che la religiosità del Manzoni è tutt'altro che pacifica e trionfalistica come si pensava un tempo - è certamente correttiva di tante letture del passato, remoto e recente.

$\mathrm{Ma}$ è veramente Dio assente quale interventista nella dimensione storica? Mariani, come abbiamo visto, dice di sì, attribuendo questa credenza ai personaggi mentre l'autore rimarrebbe ligio a una netta causalità umana. Senonché c'è qualche elemento che scalfisce questa posizione ribadita con grande insistenza. Lo dimostra un po' l'uso di termini che esprimono un giudizio di valore. Il "vecchio mal vissuto" è luciferino perché così lo vede il montanaro o perché così lo vuole il romanziere? A giudicare dalla descrizione del sollevamento popolare, caratterizzato in termini di violenza irrazionale, e alla luce di come Manzoni qualifica i non-sanguinari: "Il cielo li benedica!" (Cap. 13), si propenderebbe per quest'ultimo. Altri casi analoghi non mancano. Alcune parti della trama sollevano anch'esse qualche dubbio. Ad esempio, nel capitolo ottavo, il tocco della campana risulta mezzo di salvezza per Renzo, Lucia ed Agnese e strumento di sconfitta per i bravi. Tutto si può spiegare e si spiega in termini naturali ma non deve sfuggire il fatto che metaforicamente la campana è voce di Dio. Qualcosa di analogo si potrebbe dire per il capitolo diciassettesimo - così pieno di simbologia cristologica - capanna con paglia (mangiatoia), barcaiolo (pescatore di anime), Adda (Giordano).

Per quanto riguarda l'affermazione che la forma mentis religiosa dei personaggi è storicamente conforme a quella del Seicento riformistico, non si può non far rilevare che è stato Manzoni a farne la scelta. Dunque, non hanno completamente torto coloro che vedono in questa scelta una tattica ideologica.

In ultima analisi, si tratta però di riserve di relativamente scarso rilievo. Il contributo di Mariani, come suona il titolo, rende un Manzoni più vero rispetto a quello della critica del passato e più vero in termini epistemologici, perché in esso si delinea in modo persuasivo uno scrittore che si fa strenuo sostenitore del vero positivo della storia già al tempo dei primi Pomessi sposi.

GUIDO PUGLIESE

University of Toronto at Mississauga

Rino Caputo. Il piccolo padreterno. Saggi di lettura dell' opera di Pirandello. Roma: eUroma, 1996. Pp. 262.

Nel sempre affollatissimo panorama delle pubblicazioni pirandelliane, puntuale arriva questa sofisticata lettura testuale svolta a vari livelli, in tempi diversi e rivolta all'opera tutta di Luigi Pirandello. Quasi una "messa a punto" in chiusura di secolo o meglio di millennio sul "caso Pirandello" che tiene conto in negativo e in positivo dei contributi più autorevoli o "deplorevoli" degli ultimi decenni.

Nella sua Introduzione al testo, è lo stesso Rino Caputo a indicarci la via per una giusta "degustazione" di quello che l'autore definisce un "colloquio ermeneutico e storico-critico"(9) e che io preferisco chiamare, considerandolo a ritroso di una lettura attenta e partecipe, un personale e poietico percorso pirandelliano. Non solo di poe- 
tica, infatti, ma bensì anche di poiesi si occupa lo studioso riconoscendo a Pirandello una particolare attenzione proprio per il momento del fare artistico: è quella zona di Irespassing, è proprio quella soglia tra il qui e l'oltre il luogo in cui, pirandellianamente, avviene e si esperisce la creazione artistica. Caputo rileva infatti "la presenza costante, puntuale e, talora, quasi ossessivamente pervicace in Pirandello, del momento alto della teoresi estetica e, soprattutto, dell'intenzionalità poietica nei principali picchi compositivi ed espressivi dell'opera". (9) Questo è dunque il tipo di sguardo con cui Rino Caputo ripercorre in panoramica l'opera tutta di Pirandello, ricercando il "momento alto della teoresi estetica" ma anche quello "dell'intenzionalità poietica" a cominciare con il saggio introduttivo che dà il titolo all'intera raccolta, "Il piccolo padreterno" (18-36). In quel saggio si parla di corsi e ricorsi di blocchi concettuali ma anche testuali all'interno dell'opera pirandelliana dai testi criticoteorici a quelli più propriamente creativi: secondo la tesi dello studioso, tutta l'opera di Pirandello è integralmente "umoristica" e questo è vero anche nel "primo romanzo italiano" L'esclusa (1908), in cui la ricerca di una prossimità con il vero non è mera istanza tardo-verista (19).

Il volume di Caputo propone poi una messa in prospettiva del macrotesto pirandelliano attraverso l'analisi comparata con numerosi altri testi e macrotesti, alcuni di proficua e insolita proposizione. In "I preliminari della mente" ad esempio, ci si propone di studiare "l'operazione della mente che propone e dispone le condizioni della rappresentazione e della narrazione" analizzando "ciò che si situa tra il titolo e il testo" prendendo ad esempi il proemio di Petrarca, l'introduzione di Manzoni e la prefazione di Pirandello. In tutti e tre i casi sostiene Caputo già in ciò che si posiziona tra il titolo e il testo si ritrovano "le ragioni estetico-cognitive (poietiche e non solo di 'poetica') dell' autore: i preliminari della mente" (37). Secondo Caputo, nel caso di Pirandello, tali ragioni rivelano da un lato certo la tensione sempre esistente tra dramma e racconto, tra rappresentazione e narrazione, ma anche la fiducia dell'autore nei confronti sia della rappresentazione che della narrazione anche quando esse siano utilizzate "umoristicamente", "la poietizzazione del dramma della "commedia da fare", il racconto della storia dell'impossibilità narrativa" (51). Oppure il caso di uno studio parallelo più "solito", se si vuole, quello tra la filosofia del lontano del cannocchiale pirandelliano e la poetica pascoliana, in "Il 'cannocchiale rivoltato' e la 'poesia all'incontrario' ovvero Pirandello e Pascoli" (101-35), in cui si tracciano possibili sintonie tra il poeta e il suo recensore nonostante che l'incontro tra $i$ due autori avesse "un inizio ombroso e polemico" (105). Lo studio di Caputo germina qui dalla lettura di un foglietto manoscritto trovato tra le pagine di un libro pubblicato nel 1900 in cui Pirandello riflette argomentativamente sull'origine delle formazioni mitopoietiche di matrice greca e mediterranea. In tale foglietto si dichiara l'analogia "tra le produzioni della mente (sollecitate dal lavoro onirico e dall'intreccio tra evidenza sensibile dell"“occhio" e rielaborazione metonimica e metaforica del "pensiero") e la creazione artistica azionata dalla fantasia - nel contatto biunivoco di immaginazione e di intelletto - e coagulata nella composizione del mito" (101). Caputo sottolinea poi che nella recensione all'edizione del 1897 di Myricae, Pirandello dichiara Pascoli "il poeta capace di creare miti, il massimo rappresentante contemporaneo degli 'artefici sapienti" (107). E così che lo studioso costruisce il canale di messa in comunicazione tra Luigi Pirandello e la sua filosofia del contrario esemplificata dall'esempio del cannocchiale rovesciato del Dottor Fileno, e Giovanni Pascoli e la poetica del fanciullino. 
Tra quelli inclusi in questo nuovo volume pirandelliano, uno dei saggi più interessanti è indubbiamente quello che studia il rapporto tra la fase giovanile e la successiva produzione letteraria e, soprattutto, teatrale, studio reso possibile e legittimato dalla recente ripubblicazione in veste più organizzata e relativamente più estesa dell'epistolario sia quello curato da Providenti che quello relativo alle lettere a Marta Abba. E dunque oggi possibile approfondire lo studio del giovane Pirandello e documentare così come e quanto rilevanti fossero dall'inizio nella formazione e nell'opera dell'agrigentino centri tematici quali l'arte, la vita e il teatro (68-69). "Pirandello appare quindi, per così dire fin dall'inizio, siciliano, nazionale ed europeo e, insieme, poeta, narratore e drammaturgo"; da questa considerazione Caputo riesce poi a spingersi sino a valutare ora, sì, davvero appieno, anche quella "rivoluzione" di cui tanto si è parlato a proposito di Pirandello, una rivoluzione che spinge le sue propaggini sino all'interno dell' "attuale 'postmoderna' contemporaneità" (69).

Fra gli altri, vorrei in chiusura segnalare anche lo studio dell'umorismo nel pensiero estetico del primo Novecento poiché qui Rino Caputo traccia la mappa da un lato di una più nota sinergia tra Pirandello e il romanticismo tedesco e dall'altro di quella tra Pirandello e Baudelaire. Ammicco e sorrido all'amico-collega poiché anch'io, a suo tempo, avevo delineato la possibilità di uno studio comparato tra l'umorismo pirandelliano e il comico assoluto baudelairiano in un mio piccolo saggio del 1992. Negli ultimi tre saggi del volume Caputo da un lato offre uno studio dettagliato e sottile del grande romanzo storico di Luigi Pirandello, I vecchi e $i$ giovani, seguito poi da un'indagine puntuale del grande romanzo di Tomasi di Lampedusa, $I$ gattopardo, nel quale Caputo ravvede una notevole sintonia con le posizione espresse da Pirandello, ma anche da De Roberto e Verga, nei confronti del Risorgimento. Il volume si chiude con uno studio della letteratura nel romanzo Suo marito che viene qui visto come "snodo poietico, intriso di passato e futuro" (236) e cioè un testo narrativo che sta al centro dello sviluppo compositivo dei romanzi pirandelliani.

Questo nuovo, originale ed avvincente tentativo di ripercorrere le ragioni di poetica e di poiesi dell'autore siciliano si conclude ricordando al suo lettore un'importante ammissione fatta da Pirandello nel 1924, e cioè all'apice della sua fortuna teatrale, "Cerco una cosa sola: esprimere ciò che sento. [...] Sento, perché penso. Penso, perché sento" (252), un'affermazione con la quale si ribadisce la circolarità tra la mente e il corpo dell'opera pirandelliana, e dunque la fondamentale continuità, sempre ribadita nel testo di Caputo, tra inizio e fine del percorso pirandelliano che, secondo lo studioso, nella sua spinta rivoluzionaria, dalla fine dell'Ottocento si spinge sino alla nostra contemporanea postmodernità.

\section{MANUELA GIERI}

University of Toronto

Thomas Erling Peterson. Alberto Moravia. New York: Twayne Publishers, 1996. Pp. xii, 170.

The preparation of a critical biography presents a number of challenges with respect to crucial decisions that must be made in terms of what is to be sacrificed and what is to 